\title{
Review of: "Establishment of physiologically relevant oxygen gradients in microfluidic organ chips"
}

\author{
Eiji Kobayashi
}

Potential competing interests: The author(s) declared that no potential competing interests exist.

In this study, the authors described a strategy to achieve physiological environment of human in twochannel poly-dimethlysiloxane (PDMS) chips which enabled them to co-culture the primary human duodenal epithelium and intestinal microvascular endothelium cells in parallel channels separated by a porous membrane. When developing the chips, the authors used computer simulations that predicted oxygen concentrations and gradients in channels, and mimicked the oxygen concentration of human in PDMS chips by coating the surface of the chip with polyvinylidene chloride (PVDC) film. The cells were cultured by using their device and the authors assessed the impact on the intestinal barrier integrity in the low-oxygen environment and confirmed the barrier integrity for 72 hours.

\section{Major $\square$}

The technology for manufacturing chips reported in this manuscript is the one introduced in their previous study which was published in Nat Biomed Eng in 2019, and is the method that enables researchers to mimic the cultural conditions more easily. Although they have examined the distribution of the oxygen concentration by using both calculated values and the actual measured values and have mimicked successfully the "physiologically relevant oxygen gradients" which they discuss throughout the paper, I still feel that the study may lack novelty. Please show more clearly these attempts.

Minor $\square$

1. In Fig 3b, the sample used in the comparison is unclear.

2. In Fig. 3c, although the authors stated that the graph was shown by a heat map, it is not a heat map.

3. The authors should demonstrate the permeability as a result of the comparison of barrier integrity when cultivating endothelial cells only and co-culturing with the microbiome.

4. It appears that the period of culturing described in the previous study in 2019 has been shortened by half on the chip. Provided that the period of culturing is equal, would the equivalence be verified? 
